Document downloaded from:

http://hdl.handle.net/10251/65646

This paper must be cited as:

Seimetz, M.; Bellido, P.; Soriano Asensi, A.; Conde Castellanos, PE.; Crespo Navarro, E.; González Martínez, AJ.; Hernández Hernández, L.... (2015). Calibration and Performance Tests of Detectors for Laser-Accelerated Protons. IEEE Transactions on Nuclear Science. 62(6):3216-3224. doi:10.1109/TNS.2015.2480682.

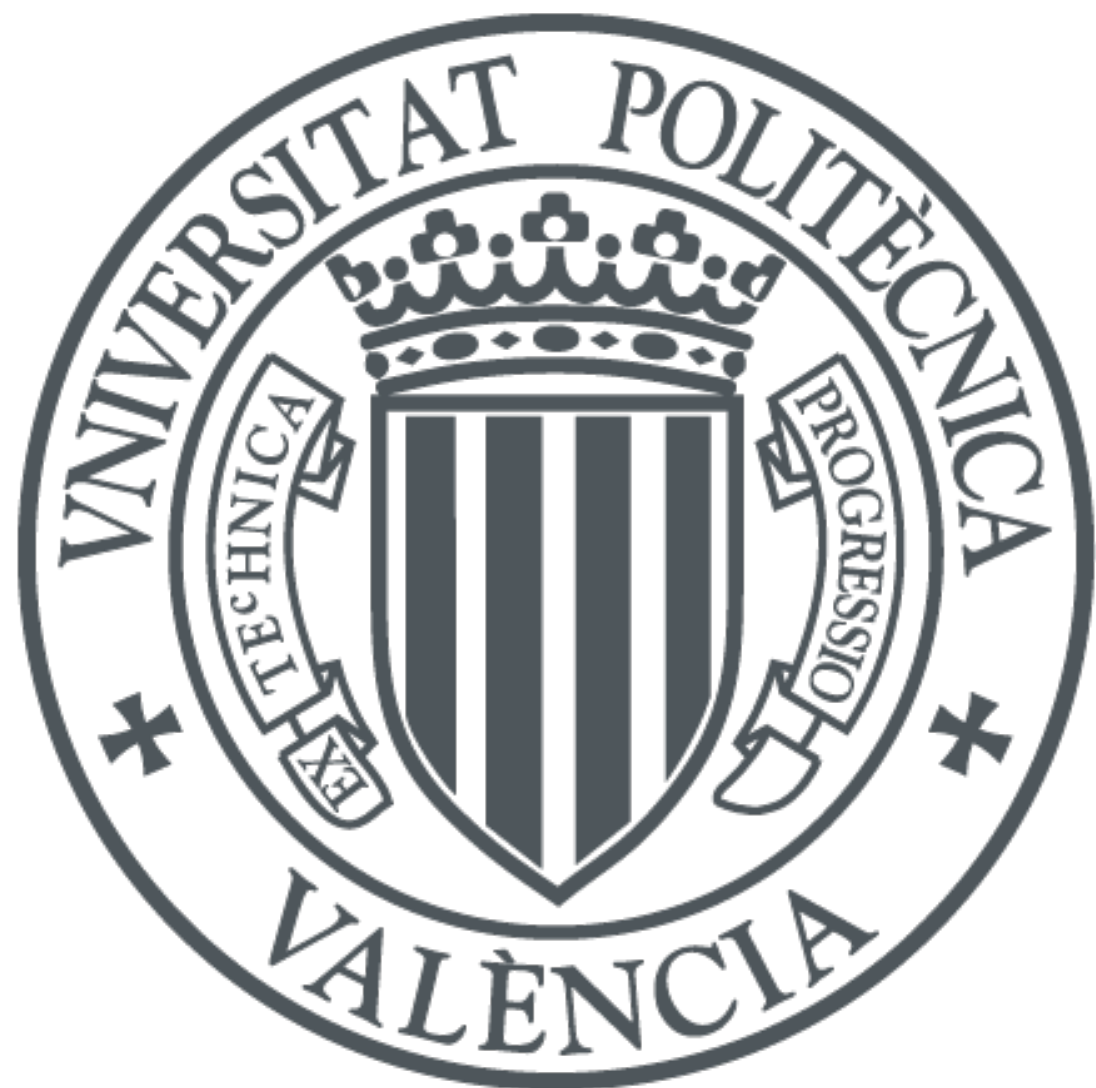

The final publication is available at

http://dx.doi.org/10.1109/TNS.2015.2480682

Copyright Institute of Electrical and Electronics Engineers (IEEE)

Additional Information

"C2015 IEEE. Personal use of this material is permitted. Permission from IEEE must be obtained for all other uses, in any current or future media, including reprinting/republishing this material for advertising or promotional purposes, creating new collective works, for resale or redistribution to servers or lists, or reuse of any copyrighted component of this work in other works." 


\title{
Calibration and Performance Tests of Detectors for Laser-Accelerated Protons
}

\author{
M. Seimetz*, P. Bellido, A. Soriano, J. García López, M. C. Jiménez-Ramos, B. Fernández, P. Conde, E. Crespo, \\ A. J. González, L. Hernández, A. Iborra, L. Moliner, J. P. Rigla, M. J. Rodríguez-Álvarez, F. Sánchez, \\ S. Sánchez, L. F. Vidal, and J. M. Benlloch
}

\begin{abstract}
We present the calibration and performance tests carried out with two detectors for intense proton pulses accelerated by lasers. Most of the procedures were realised with proton beams of 0.46-5.60 MeV from a tandem accelerator. One approach made use of radiochromic films, for which we calibrated the relation between optical density and energy deposition over more than three orders of magnitude. The validity of these results and of our analysis algorithms has been confirmed by controlled irradiation of film stacks and reconstruction of the total beam charge for strongly non-uniform beam profiles. For the spectral analysis of protons from repeated laser shots, we have designed an online monitor based on a plastic scintillator. The resulting signal from a photomultiplier directly measured on a fast oscilloscope is especially useful for time-of-flight applications. Variable optical filters allow for suppression of saturation and an extension of the dynamic range. With pulsed proton beams we have tested the detector response to a wide range of beam intensities from single particles to $3 \times 10^{5}$ protons per 100 ns time interval.
\end{abstract}

Index Terms-Proton detectors, Organic scintillators, Particle beam measurements

\section{INTRODUCTION}

$\mathbf{P}$ ROTON and ion acceleration by ultra-short, ultra-intense laser pulses has become a very active research field throughout the last decade. The spectral distribution of protons, accelerated at several high-power laser facilities worldwide, strongly varies with laser parameters such as the pulse power, focused intensity, prepulse duration, and contrast, as well as the target material and thickness [1]. A deeper understanding of the underlying mechanisms in different intensity regimes [2] and the optimisation of experimental conditions

Manuscript received January 14, 2015; revised June 30, 2015; accepted September 15, 2015. Project funded by the Spanish Ministry of Economy and Competitiveness and co-funded with FEDER's funds within the INNPACTO 2011 program, Grant No. IPT-2011-0862-900000. This work was supported by the Spanish Plan Nacional de Investigación Científica, Desarrollo e Innovación Tecnológica (I+D+i) under Grant No. TEC 2013-48036-C3-1-R and the Valencian Local Government under Grants PROMETEOII/2013/010 and ISIC 2011/013. A.J. González is financed by CSIC with a JAE-Doc contract under Junta de Ampliación de Estudios program, cofinanced by the European Social Fund.

M. Seimetz, P. Bellido, A. Soriano, P. Conde, E. Crespo, A. J. González, L. Hernández, A. Iborra, L. Moliner, J. P. Rigla, M. J. Rodríguez-Álvarez, F. Sánchez, S. Sánchez, L. F. Vidal, and J. M. Benlloch are with Instituto de Instrumentación para Imagen Molecular (I3M), CSIC - Universidad Politécnica de Valencia - CIEMAT, Camino de Vera s/n, Ed. 8-N-1, 46022 Valencia, Spain.

J. García López, M. C. Jiménez-Ramos, and B. Fernández are with Centro Nacional de Aceleradores (CNA), U. Sevilla - J. Andalucía - CSIC, Avda. Thomas Alva Edison 7, 41092 Sevilla, Spain.

*Corresponding author. Contact: mseimetz@i3m.upv.es. in view of possible applications [3] are key aspects of current research activities.

The spectral analysis of laser-accelerated protons and ions requires the almost instantaneous detection of large numbers of particles. Some types of detectors have been applied in several independent experimental setups [4]. These comprise passive media like image plates, CR-39 nuclear track detectors, and radio-sensitive films [5], [6] which are convenient for their flexibility, robustness, and simple preparation, but require post-processing after every laser shot. For repeated measurements detectors with real-time readout are preferrable, such as Thomson parabola spectrometers [7], [8], silicon-based pixel detectors [9], and systems based on scintillators [10][12] or scintillating fibres [13]. A time-of-flight method for the measurement of proton and ion energies with Faraday cups and semiconductor detectors has been presented in [14].

In this work, we present the calibration and performance tests of proton detectors designed for laser-plasma experiments. These detectors permit the characterization of proton spectra up to energies around $15 \mathrm{MeV}$, accelerated by lasers with 10-200 TW pulsed power. The present types of detectors are radiochromic films (RCF) and a specially designed online monitor based on a plastic scintillator. Their basic outline will be justified in section III by the expected experimental conditions. In section [III we describe our choice of RCF and the calibration of their response to a range of radiation doses deposited by proton beams under controlled conditions. In addition, we have tested the reconstruction of proton spectra by irradiating a RCF stack and measuring the response of each layer. The setup of the online monitor, together with a series of measurements over a broad dynamic range, will be presented in section IV

\section{Design CONSIDERATIONS}

Published results on laser-accelerated protons reveal large differences in terms of absolute numbers of particles and their energies. These reveal a strongly non-linear dependence on several experimental parameters, implying large uncertainties in the prediction of the expected outcome of a future setup. In our prospective interval of focused laser intensities $\left(10^{18}\right.$ $10^{19} \mathrm{~W} / \mathrm{cm}^{2}$ ), protons with maximum energies between 1.3 and $30 \mathrm{MeV}$ have been reported [1]. Their spectra are typically broad, falling exponentially from the lower detection limit (below $1 \mathrm{MeV}$ ) to a sharp high-energy cutoff. Particle numbers may be as large as $10^{11}$ for a single laser shot [15] although 
much lower quantities $\left(10^{4}\right)$ have also been found, especially for gaseous targets or other materials with low density [16]. The acceleration time of these protons is comparable to the duration of the laser pulse (of the order of 100 femtoseconds), far below the response time of any particle detector. They are spatially concentrated around an axis, which is approximately oriented along the normal direction at the rear side of the laser target.

Our choice of particle detectors for the characterisation of protons emitted in single laser shots is based on these findings. Similar techniques have been used in previous laseracceleration experiments. Radiochromic films provide a very flexible and precise means to image the spatial distribution of particles at any distance from the target. Their optical density after irradiation is related to the local dose deposition and can be used for the reconstruction of the proton kinetic energy after proper calibration (see section IIII. Protons above $3 \mathrm{MeV}$ traverse a single RCF with a thickness around $100 \mu \mathrm{m}$ and can be detected in subsequent foils. Thus, a stack of various layers provides additional information on the spectral distribution of the particles.

Radiochromic films are of single use only; an area once irradiated cannot be reused for further measurements (unless several laser shots are accumulated to increase statistics). Moreover, they must be retrieved from the experimental setup (inside a vacuum chamber) and post-processed to obtain quantitative results on the deposited energy. For a large number of laser shots, a detector providing real-time information is preferable. To this purpose, we have designed an online monitor based on a single plastic scintillator with $25 \times 25 \mathrm{~mm}^{2}$ active area coupled to a photomultiplier (PMT) through optical fibres (section IV]. This unsegmented configuration does not offer spatial information on the particle distribution. Instead, a good relation between energy deposition and pulse amplitude is provided, both for single protons as well as for overlapping signals of large numbers of particles, as those observed in previous laser-acceleration experiments [17]. The PMT anode pulse is displayed on a fast oscilloscope and stored for subsequent analysis.

The online monitor may be mounted closely behind the laser target or at $1.5-2 \mathrm{~m}$ distance. The detection time after the laser pulse is directly related to the proton energy (time-offlight method, TOF) [11], [17]. In our case, the projected range of kinetic energies $(0.5-15 \mathrm{MeV})$ corresponds to flight times between 153 and $30 \mathrm{~ns}$ at $1.5 \mathrm{~m}$ target distance, respectively. Since spectra of laser-accelerated protons generally are broad, they form a single overlap pulse of approximately $120 \mathrm{~ns}$ length. In order to obtain a reasonable energy resolution, the duration of single-particle pulses must be much shorter than the time-of-flight differences. Organic scintillators are inherently fast, with decay times of a few nanoseconds, and thus fulfill this condition.

At $1 \mathrm{~m}$ target distance the angular coverage of our scintillator is approximately $0.5 \mathrm{msr}$. The detector will usually be placed at the position of highest proton flux, as previously determined with the RCF, corresponding to the highest particle energies as well. From laser acceleration experiments similar to our projected conditions (10-200 TW pulsed power), a max- imum proton flux of $10^{7}-10^{8} / \mathrm{MeV} / \mathrm{msr}$ per shot is expected at this position [1]. This has important implications for the use of a scintillator-based detector with PMT readout, which may experience saturation effects. These can originate both from high local ionisation densities inside the scintillator material and from the breakdown of the anode voltage at high PMT output currents. It is well known that the ionisation densities of electrons, protons, and ions in the scintillator material lead to different light output at similar deposited energies [18]. This is, however, not a major concern for the present study restricted to proton detection. In principle, at fixed particle energy the number of scintillation photons will be proportional to the number of incident protons, unless the ionised volumes of neighbouring particles start to overlap. Typical track diameters of 1-10 MeV protons in polymeres are of the order of 5$20 \mu \mathrm{m}$, as is known from measurements with CR-39 [19]. Thus proportionality in light output may be assumed up to about $6 \times 10^{6}$ uniformly distributed protons within our $25 \times 25 \mathrm{~mm}^{2}$ active area, close to the expected maximum flux. In contrast, the dynamic range of the PMT at constant high voltage is limited to about one order of magnitude in detected photons, making its saturation more critical for our application. In order to adjust the number of scintillation photons reaching the PMT according to the incident particles, we provide the possibility to insert different neutral-density filters between the optical fibres and the PMT entrance window. The dynamic range can thus be extended over several decades, starting from the detection of single particles.

The relatively large thickness of our scintillator $(5 \mathrm{~mm})$ as compared to previous experiments $[10]-[12]$ has been chosen to ensure that protons in the projected energy range will be stopped. Thus, for single particles a unique relation between pulse height and proton energy is provided. For broad spectral distributions our choice pronounces the contribution of particles at high energies. This may be advantageous for the measurement of the maximum energy of exponentially decaying spectra.

As mentioned, radiochromic films and the online monitor provide distinct capabilities for the characterisation of laseraccelerated protons, each of them with their own advantages and restrictions. For example, the angular distribution of the proton flux may straightforwardly be imaged by RCF, while an unsegmented scintillator may provide only very limited information unless its position, or the aperture of an additional collimator, are varied over a major number of laser shots. Nevertheless, the two detector types can be applied for mutual cross checks. Spectral information can be obtained from both. During our performance tests with the online monitor, we applied the RCF to control the intensity of pulsed proton beams (see section IV-B3).

\section{RADIOCHROMIC FILM}

Radiochromic films are widely used for dosimetry purposes, e.g. in radiotherapy. Contrary to photographic emulsions or CR-39, RCF do not exhibit good response to single particles. Instead, their optical density varies when exposed to major dose depositions starting from some cGy for films with thick 
active layers such as EBT3 [20], or above 10 Gy approximately for thinner ones [21]. Many types of RCF are made in a sandwich structure with a radiation sensitive substrate between two layers of a stable, transparent polymere. For the detection of protons below $1 \mathrm{MeV}$, with very limited range inside solid materials, it is preferrable to provide an active layer directly at the entrance surface, as for our choice of product, Gafchromic $^{\mathrm{TM}} \mathrm{HD}-\mathrm{V} 2$, with a $12 \mu \mathrm{m}$ thick sensitive polymere on a base of $97 \mu \mathrm{m}$ of polyester [22]. The only postprocessing necessary for this kind of film is high-resolution scanning in transmission mode (48 bit colours, $300 \mathrm{dpi}$ ). The net optical density of an image pixel, $\mathrm{OD}_{\text {net }}$, is obtained from the grey value in the red colour channel, $g_{\text {red }}$, and the background, $b_{\text {red }}$, as

$$
\mathrm{OD}_{\text {net }}=\log _{10} \frac{b_{\mathrm{red}}}{g_{\text {red }}} .
$$

We have evaluated the systematic errors of this procedure in test measurements prior to data taking with protons. The relative deviation after several repetitions of the same scan (reproducibility) and after repeating the procedure on different parts of the scanner surface (uniformity) has been found to be below $1 \%$ [23].

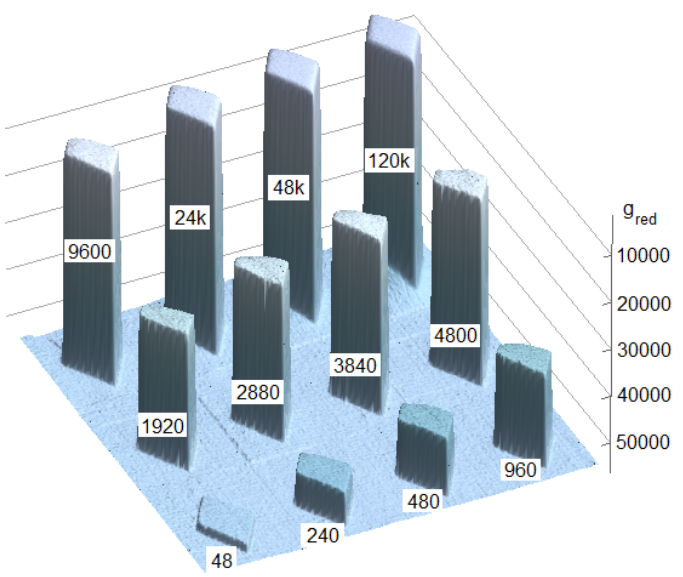

Fig. 1. Grey levels of radiochromic film HD-V2 after different irradiation times at constant proton beam current. The height of the prisms corresponds to pixel values, $g_{\text {red }}$, with a background of $b_{\text {red }} \simeq 55000$. Data taken with a $4 \mathrm{MeV}$, continuous proton beam. The labels indicate the total beam charge collected inside the irradiated areas (in pC).

\section{A. Calibration}

In order to relate the optical density to the dose deposited in the active layer, we have calibrated the RCF with monoenergetic proton beams from the $3 \mathrm{MV}$ tandem accelerator at the Spanish National Accelerator Center (CNA) at Seville. Three different series of overlapping dose intervals have been recorded, two of them with continuous beams at $4 \mathrm{MeV}$ $(60 \mathrm{pA})$ and $1.5 \mathrm{MeV}(250 \mathrm{pA})$ proton energy. The third one was taken in pulsed mode at $1.5 \mathrm{MeV}$ using $100 \mu$ s long intervals at $1 \mathrm{kHz}$ repetition rate (for details of the pulsed mode operation see section IV-B). The irradiated area was delimited by a rhombic collimator and kept constant throughout each series. Irradiation times varied between 1 and $1000 \mathrm{~s}$ to cover a broad range of deposited doses (Fig. 1). While the grey level of the rhombic area is uniform for the $1.5 \mathrm{MeV}$ data, at $4 \mathrm{MeV}$ beam energy we have observed considerable deviations of the grey values within the exposed area due to a non-homogeneous proton flux density (up to $10 \%$ approximately).

The proton flux was monitored by a current integrator (Brookhaven Instruments Corporation Model 1000C) collecting the beam charge, $Q_{\mathrm{CI}}$, accumulated on an electrically isolated, metallic target holder on which the RCF were mounted. To suppress the secondary electrons produced by the proton beam, the sample holder was biased to $+300 \mathrm{~V}$ using a battery. The accuracy of the beam current measurements was about $2 \%$. From $Q_{\mathrm{CI}}$ and the known beam energy, we calculated the energy deposited in the sensitive film layer. The energy loss of protons as a function of kinetic energy, required for this procedure, has been calculated from a simulation program (SRIM) [24] using the chemical composition of HD-V2 (active layer $\left(1.2 \mathrm{~g} / \mathrm{cm}^{2}\right): 27.9 \% \mathrm{C}, 58.4 \% \mathrm{H}, 11.7 \% \mathrm{O}, 0.6 \% \mathrm{Li}, 0.1 \%$ $\mathrm{N}, 0.5 \% \mathrm{Na}, 0.6 \% \mathrm{Cl}, 0.3 \% \mathrm{Al}$; polyester $\left(1.35 \mathrm{~g} / \mathrm{cm}^{2}\right): 45.5 \%$ $\mathrm{C}, 36.4 \% \mathrm{H}, 18.2 \% \mathrm{O}$; data provided by the manufacturer) [22]. We have thus obtained the calibration data displayed in Fig. 2, relating the optical density with the energy deposition per area, $E_{d} / A$. The irradiations at different beam energies and operation modes have given consistent results.

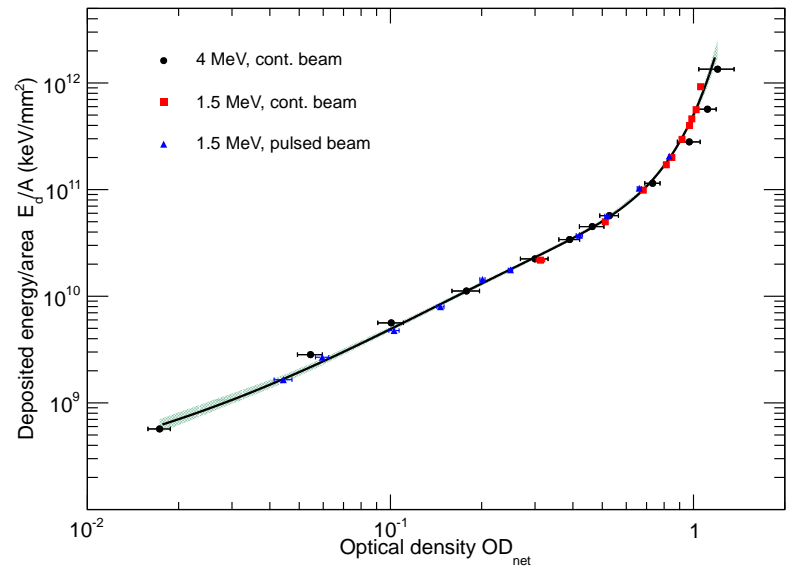

Fig. 2. (color on-line) Calibration data for RCF under different proton beam conditions. The line represents a fit to the three data sets.

The largest uncertainty of the single data points stems from the inhomogeneity of the proton beam profile. The horizontal error bars of Fig. 2 represent the total interval of $\mathrm{OD}_{\text {net }}$ of each data point. The precision of the scan, as stated above, is better than $1 \%$. The error of the energy deposition per area is dominated by the charge measurement (2\%) and the model uncertainty of SRIM (4\%). The uncertainties in beam energy $(0.1 \%)$ and measured area size are negligible.

In order to obtain a continuous calibration curve for the probed range of energy depositions between $E_{d} / A=6 \times 10^{8}$ and $2 \times 10^{12} \mathrm{keV} / \mathrm{mm}^{2}$, the data points of all three sets have been fitted to an exponential function,

$$
\frac{E_{d}}{A}=1 \frac{\mathrm{keV}}{\mathrm{mm}^{2}} \cdot e^{p_{0}+p_{1} x^{0.5}+p_{2} x+p_{3} x^{2}},
$$


with $x \equiv \mathrm{OD}_{\text {net }}$. The best fit $\left(\chi^{2} / \mathrm{ndf}=41.8 / 28\right)$ to this purely empirical formula has been obtained with the following parameters: $p_{0}=18.15 \pm 0.12, p_{1}=17.85 \pm 0.60$, $p_{2}=-15.47 \pm 0.80, p_{3}=6.41 \pm 0.34$. Suppression of any of these coefficients results in an increase of $\chi^{2}$ by a factor 15 or more. The $95 \%$ confidence level of the fit is indicated as shaded area in Fig. 2. It corresponds to approximately 4-5\% relative error in $E_{d} / A$ over a wide range of optical densities, except the regions close to the upper and lower limits. This precision is guaranteed only for the RCF material used in our calibration. An additional restriction is imposed by batch-tobatch variations of the radiochromic film sensitivity (up to $10 \%$ as stated by the manufacturer). In the calibrated interval, the absolute number of protons per area ranges from $4 \times 10^{6}$ to $9.6 \times 10^{9} / \mathrm{mm}^{2}$.

\section{B. Performance tests}

When protons of several $\mathrm{MeV}$ hit a stack of radiochromic films, the grey value of each foil depends on the energy deposited in its active layer. This is illustrated in Fig. 3 . where the energy deposition is displayed as a function of depth inside an RCF stack for single protons of different energies. The relation between the optical density and the particle energy is highly non-linear. A $3 \mathrm{MeV}$ proton deposits a large part of its kinetic energy in the sensitive part of foil 2, while one of $4 \mathrm{MeV}$ only produces a small signal in foil 2 and a proton of $2 \mathrm{MeV}$ is absorbed in the first layer. In addition, saturation effects at very high ionisation densities imply a non-proportionality between optical density and deposited energy close to the Bragg peak [25], which has been considered in the analysis. In order to test the precision of the underlying depth-dose calculations, carried out with SRIM, we have irradiated a four-film stack with a proton beam of $5.5 \mathrm{MeV}$. The energy deposition in each layer (integrated over the irradiated area of approximately $8 \times 8 \mathrm{~mm}^{2}$ ) has been reconstructed from the optical density and the calibration curve obtained previously. Our results compare very well with the expectations (Fig. $4(\mathrm{a})$ ); the mean value of the relative deviation between measured and calculated energies in each layer, $\left|E_{d}-E_{d}^{\mathrm{SRIM}}\right| / E_{d}$, is $2.1 \%$. Here the experimental errors in $E_{d}$ stem from the uncertainty of the calibration curve (approximately 5\% for a wide range of OD) and the proton flux measurement $(2 \%)$, whereas the calculated values have a $4 \%$ uncertainty from SRIM [24].

In our laser-acceleration experiments, we will most likely find broad proton spectra, i.e. mixtures of many particle energies. In order to simulate the detector response of our RCF to such conditions, we have irradiated a second four-film stack with protons of five different energies (Fig. 4(b)). The stack has been exposed to a $2.0 \mathrm{MeV}$ beam until a predefined charge limit was reached on the current integrator. Then the procedure was repeated under the same conditions (RCF position, collimator aperture, and accumulated charge limit) with $3.03,4.08,4.98$, and $5.57 \mathrm{MeV}$ protons, respectively. Note that in this case the optical density of layer 2 is the largest one, due to the Bragg peak of the $3.03 \mathrm{MeV}$ protons. Here, the mean deviation between measured and calculated

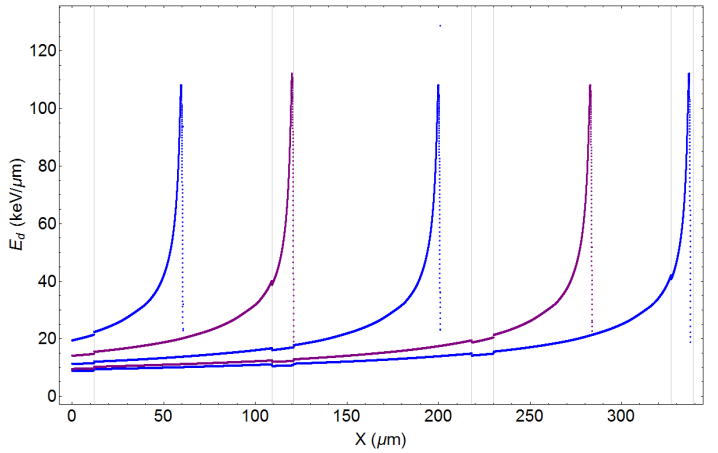

Fig. 3. Calculated depth dose profiles (Bragg peaks) for 2.0, 3.0, 4.0, 5.0, and 5.5 MeV single protons (from left to right). Data obtained with SRIM.

energies is $4.9 \%$. All pairs of data points are consistent within their uncertainties.

(a)

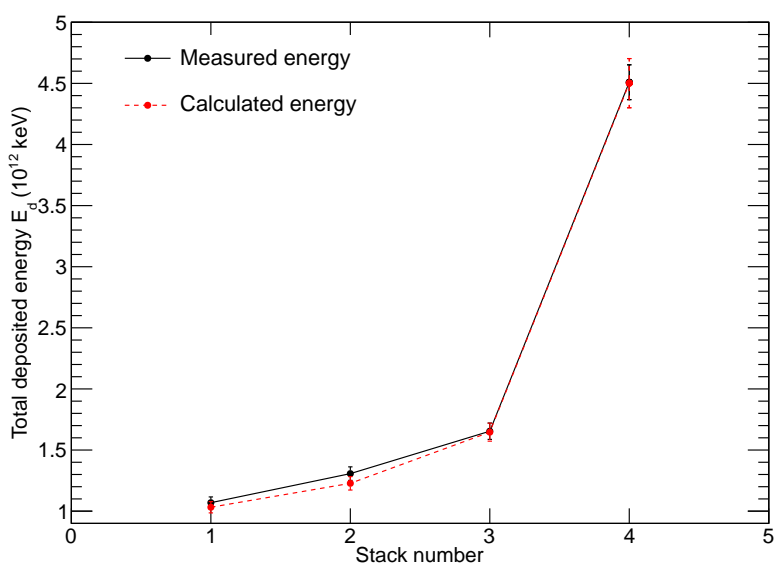

(b)

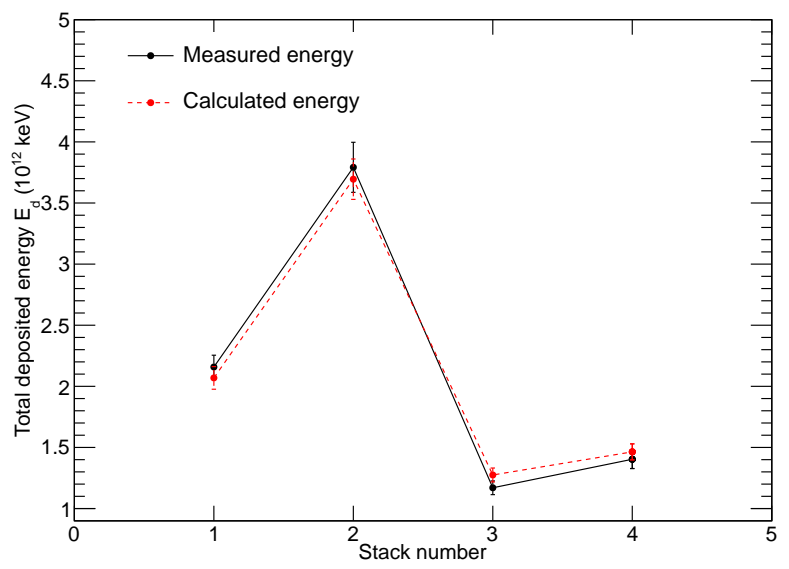

Fig. 4. Measured energy depositions in a stack of four RCF, compared to SRIM calculations. (a) Single proton energy $(5.5 \mathrm{MeV})$. (b) Subsequent irradiation with five different energies $(2.00,3.03,4.08,4.98$, and $5.57 \mathrm{MeV})$.

Finally, we have tried the reconstruction of particle numbers for very non-uniform beam profiles (Fig. 5). These tests have been carried out with a single RCF layer exposed to a $1.5 \mathrm{MeV}$ proton beam to cross-check the measurements of 
pulsed beam currents with a current integrator (see section IV-B3). The RCF, mounted on a controllable target holder, was moved to the beam position several times within the test series to irradiate previously unused areas during time intervals of 5,10 , and $20 \mathrm{~s}$. We have obtained the integral grey value after scanning and, at known beam energy, the total deposited energy and charge, $Q_{\mathrm{RCF}}$. The results are very consistent with the current integrator readout, $Q_{\mathrm{CI}}$. For 17 measurements, we have calculated the relative deviation, $\left|Q_{\mathrm{CI}}-Q_{\mathrm{RCF}}\right| / Q_{\mathrm{CI}}$, and found a mean value of $4.1 \%$. This good agreement demonstrates the validity of our calibration to obtain absolute particle numbers from non-uniform images, similar to those expected from laser-accelerated protons.

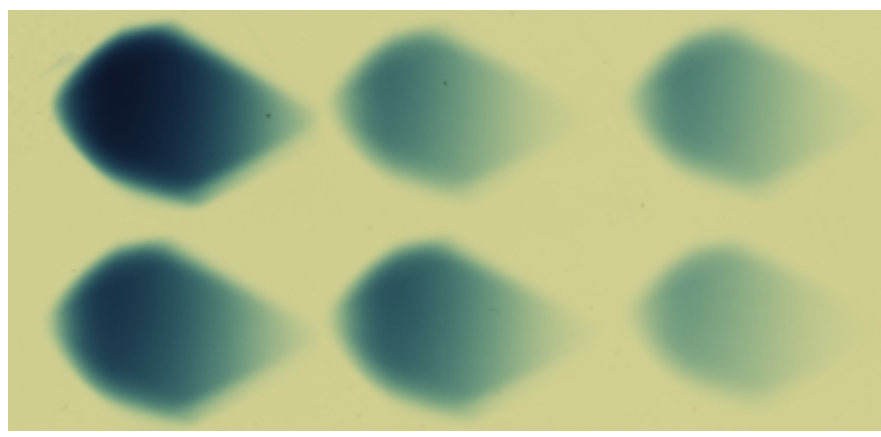

Fig. 5. Non-uniform beam profiles observed with a single RCF layer at 1.5 $\mathrm{MeV}$ proton energy.

\section{ONLINE MONITOR}

Organic scintillators are standard tools of nuclear and particle physics [26]. With a very short decay time of a few nanoseconds and in combination with fast detectors for scintillation photons, such as PMTs or SiPMs, they are frequently used for timing measurements. Their most common application is the detection of single particles. In laser-plasma interactions, large numbers of particles are released in time periods of several tens or hundreds of femtoseconds. Even when such ultra-short pulses of protons are spread out over a flight path of 1-2 m due to different particle momenta, they will most likely produce an overlap signal inside a scintillator. Nevertheless, this type of detectors has been successfully applied for the measurement of energy spectra of laser-accelerated protons [10], [11]. Our design, as motivated in section II] tends to offer maximum flexibility to obtain the proton spectral distribution, especially at the high-energy limit, over a wide range of particle numbers. The scintillator is also capable of detecting photons and relativistic electrons originating from the laser-plasma interaction. These particles will be clearly separated from protons and ions due to their very different flight time.

\section{A. Setup}

The sensitive part of our online monitor is made of a common plastic scintillator material (NE102A) with an emission maximum at $423 \mathrm{~nm}$ and $2.4 \mathrm{~ns}$ decay time [26]. It has a $25 \times 25 \mathrm{~mm}^{2}$ active area and $5 \mathrm{~mm}$ thickness, sufficient to stop protons up to $22.5 \mathrm{MeV}$. The scintillator is wrapped with $2 \mu \mathrm{m}$ thick aluminised mylar for protection from external light at minimum energy loss and mounted in a protective housing (Fig. 6). Five optical fibres (Eska ${ }^{\mathrm{TM}}$ CK-120, $3 \mathrm{~mm}$ diameter) provide a mechanically flexible connection between the scintillator and the PMT. With their large diameter, they cover $28 \%$ of the scintillator output face. In previous tests, we found a better transmission efficiency for this configuration than for bundles of thinner fibres with the same total cross section. In order to achieve a uniform detection efficiency in the part of the scintillator close to the fibres, an intermediate light guide has been designed, consisting of a rectangular block of PMMA and five tapered cones [27].

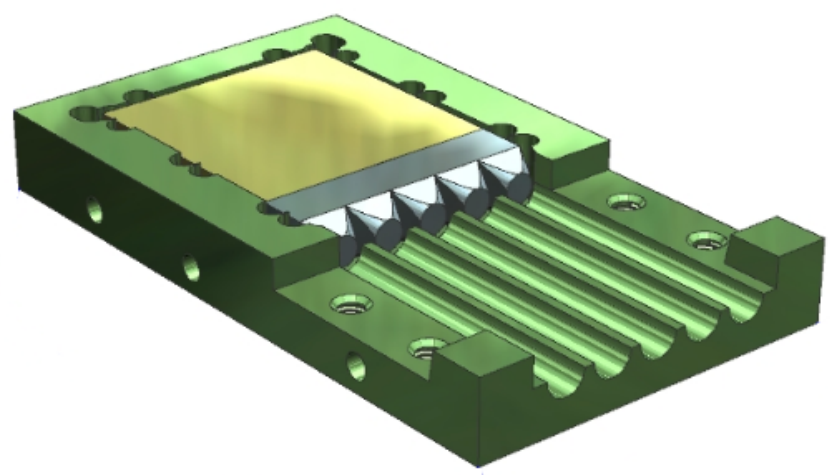

Fig. 6. Plastic scintillator and light guide in the protective housing.

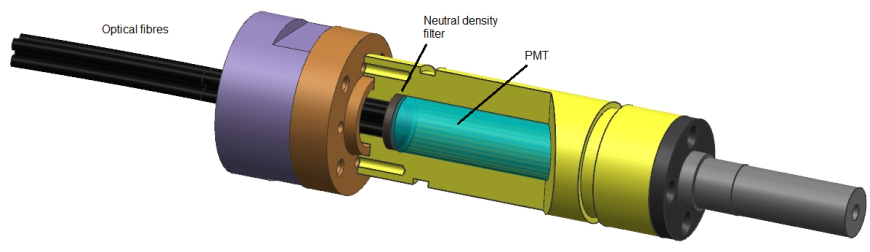

Fig. 7. Photomultiplier connected to optical fibres. A neutral density filter can be mounted between the fibres and the PMT entrance window.

The five optical fibres are dry-coupled to the entrance window of a single photomultiplier tube (Hamamatsu R647, $10 \mathrm{~mm}$ active diameter, with an E849-35 socket assembly). Optical filters can easily be introduced between the fibres and the PMT to adjust the dynamic range (Fig. 7). In our performance tests, we have worked without filter for the detection of single particles, and with neutral density filters of OD1-3 for many-particle pulses. The PMT analogue output current is recorded without further amplification on an oscilloscope with $1 \mathrm{GHz}$ bandwidth (Agilent Technologies MSO 7104B).

\section{B. Test procedures and results}

The online monitor is intended to operate over a wide range of proton beam intensities and to provide quantitative information on the particle numbers and energies. In the single-particle regime, the operating parameters are adjusted to guarantee that a wide range of proton energies can be resolved (especially below $1 \mathrm{MeV}$ ). The transition to bunches of multiple, overlapping particles, which resemble the conditions of 
laser acceleration experiments, is not trivial since the detector response, especially of the PMT, may be non-linear. The aim of our test series is to determine the range of intensities where signal proportionality can be assumed, and to demonstrate the validity of the use of intermediate optical filters.

1) Response to single particles: The first topic we have addressed in our performance tests is the response of the scintillator to single particles, starting with different radioactive sources throughout a wide range of energies. At a given PMT high voltage setting $(910 \mathrm{~V}$, at the lower end of the recommended range), we have clearly observed pulses of $\gamma$ emitters $\left({ }^{137} \mathrm{Cs},{ }^{22} \mathrm{Na},{ }^{60} \mathrm{Co}\right)$ with photon energies between $E_{\gamma}=0.511$ and $1.33 \mathrm{MeV}$, and of $\beta$ sources $\left({ }^{204} \mathrm{Tl}\right.$ and ${ }^{90} \mathrm{Sr}$ ) with maximum energies $E_{\beta}=0.764$ and $2.28 \mathrm{MeV}$, respectively. The identification of lower-energy photons $\left({ }^{153} \mathrm{Gd}\right.$ and ${ }^{57} \mathrm{Co}, E_{\gamma}=0.1 / 0.12 \mathrm{MeV}$, respectively) required PMT voltages above $1100 \mathrm{~V}$. The pulses show a very good signalto-noise ratio and a width of approximately 6 ns FWHM (Fig. 8,a)). This is far below the expected length of multiparticle pulses from laser accelerated protons (120 ns, see section II). (a)

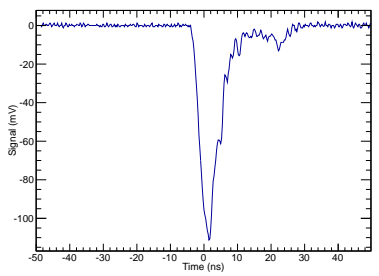

(b)

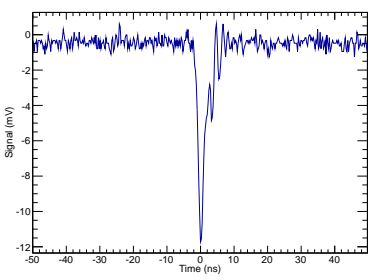

Fig. 8. Oscilloscope pulses of a $\gamma$ source (a) and a proton at $0.46 \mathrm{MeV}$ beam energy (b).

A second test series has been performed at the $3 \mathrm{MV}$ tandem accelerator of CNA. At the lowest possible, continuous beam currents (below $1 \mathrm{pA}$ ), separate pulses from single protons have been identified on the oscilloscope. At constant PMT voltage $(910 \mathrm{~V})$ we have observed proton signals within the entire available energy range $\left(E_{p}=0.46-5.6 \mathrm{MeV}\right)$. At $E_{p}=0.46 \mathrm{MeV}$, typical pulse amplitudes are around $10 \mathrm{mV}$, but clearly above the noise level (Fig. 8(b)). The clear observation of protons at far below $1 \mathrm{MeV}$ is important for our projected application, because it demonstrates that these particles pass through the protective mylar film and that their scintillation light, emitted within a few microns close to the entrance surface, is sufficient to produce visible signals on the oscilloscope.

2) PMT saturation at long pulses: In the detection of multiple protons within a time interval of the order of $100 \mathrm{~ns}$, the height of the overlap signal may not be simply proportional to the single-particle pulse and the number of protons, due to saturation effects, mainly of the PMT. In our third test, we have studied the response of the photomultiplier to long input signals at constant intensity, originating from a light pulser (model BNC 6010G, Berkeley Nucleonics Corporation). $500 \mathrm{~ns}$ long pulses at $450 \mathrm{~nm}$ wavelength with different light pulse amplitudes were injected directly onto the PMT (not via the scintillator and fibres). The PMT anode pulse was observed on the oscilloscope with $50 \Omega$ input impedance. A rectangular signal is found for pulse amplitudes up to $250 \mathrm{mV}$, and the rise time $(15 \mathrm{~ns})$ and fall time $(50 \mathrm{~ns})$ quoted for the pulse generator [28] are fairly reproduced (Fig. 9]. Since the pulse duration is much longer than the time-of-flight interval of detectable protons, we are confident that up to $250 \mathrm{mV}$ any variations in observed pulse heights reflect true patterns of the particle intensity and that they are not artefacts of the photomultiplier response. At $300 \mathrm{mV}$ initial pulse height, the signal shows a negative slope after $300 \mathrm{~ns}$ approximately, and a signal with $350 \mathrm{mV}$ initial level decreases after $150 \mathrm{~ns}$. As a consequence, for particle detection the neutral density filters have to be adapted in the way to guarantee a maximum pulse height below $250 \mathrm{mV}$.

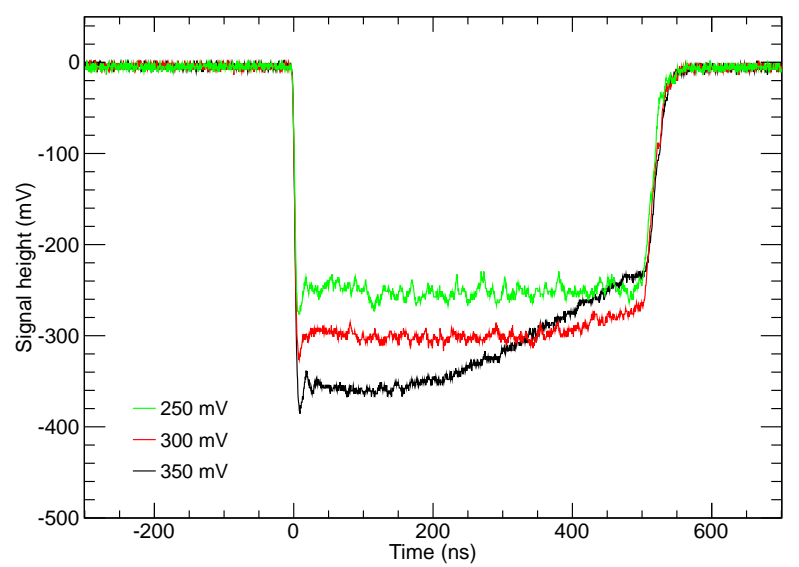

Fig. 9. (color on-line) PMT output pulses induced by a light pulse generator.

The forth series of performance tests has been carried out at the CNA linear accelerator, this time using a pulsed proton beam. To this purpose, a beam kicker has been installed between the proton source and the tandem. The kicker consists in a pair of metal plates, on which a high voltage can be applied in quick switching mode to deflect the low-energy protons. The bunch length and repetition rate are controlled by an input pulse generator. Fig. 10 illustrates the PMT output signals for different bunch lengths with an initial pulse height of $200 \mathrm{mV}$, i.e. close to the previously observed saturation level. A 500 ns proton bunch produces a stable detector response. At $1 \mu$ s length a slight drop can be recognized, and for bunches of several $\mu$ s, the PMT voltage breaks down. We conclude that the response of the online monitor to multiproton pulses is stable up to signal levels around $200 \mathrm{mV}$ and within time intervals up to $1000 \mathrm{~ns}$, which is much more than the projected regime of laser-accelerated protons.

The pulse shape visible in Fig. 10 shows a short prepulse within the first $100 \mathrm{~ns}$, which is not an artifact of the detector, but rather results from the interaction of the kicker with the low-energy proton beam. Throughout the series of measurements reported in the following sections, nominal bunch lengths of $500 \mathrm{~ns}$ at $1 \mathrm{kHz}$ repetition rate have been generated. However, the oscilloscope signals revealed different time structures depending on the daily beam optimisation; the 

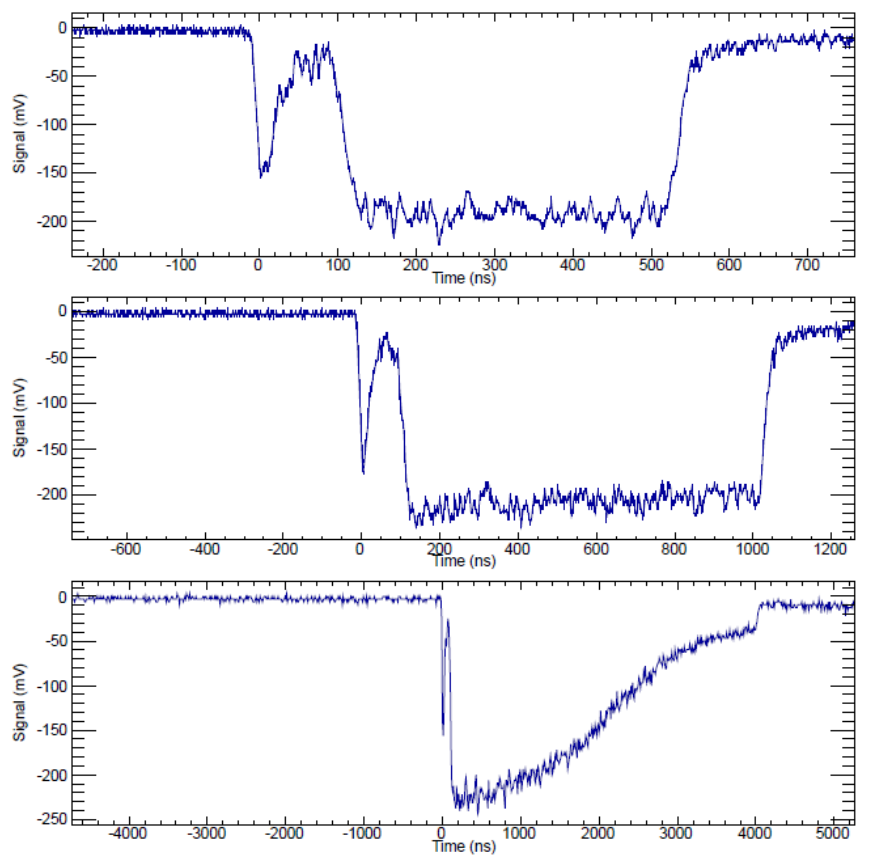

Fig. 10. PMT output signals from proton pulses of different widths: $500 \mathrm{ns,}$ $1 \mu \mathrm{s}$, and $4 \mu \mathrm{s}$ (from top to bottom; note different time scales).

beam intensity was not always constant over the full $500 \mathrm{~ns}$ period. For the quantitative analyses (e.g., the observed pulse height as a function of beam intensity), we have made use of time intervals in which the oscilloscope signal could be considered approximately stable.

3) Intensity variation of multiple-proton bunches: The principal aim of the fifth kind of measurements was to simulate the detection of multiple-particle bunches over a wide range of intensities. At constant proton energy, the beam current on the scintillator was varied in several successive series, each of them with a different optical filter. The scintillator was placed behind a rhombic collimator with a maximum aperture of approximately $1 \mathrm{~cm}^{2}$, smaller than the beam diameter. In this way the proton beam illuminated a major part of the detector surface and the beam intensity could be controlled merely by variation of the collimator aperture.

The scintillator was mounted on a metallic, movable target holder, which was electrically isolated. Before each irradiation of the scintillator an aluminium plate, covered with phosphor powder and skrewed onto the same target holder, was moved into the beam position to control the size of the irradiated area and to measure the beam current on the target via a current integrator (CI). These current measurements, which were crucial for the purpose of our experiment, were challenged by several factors. The nominal low range limit of the $\mathrm{CI}$ is $0.6 \mathrm{pA}$. In practice, measured values below $10 \mathrm{pA}$ are less precise than those at higher currents (standard precision: 2\%), because of electronic noise. In continuous mode (kicker switched off), the intensities applied were above this critical interval, from $42 \mathrm{pA}$ to $390 \mathrm{nA}$. However, due to the above-mentioned interaction of the kicker with the beam, the absolute intensity in pulsed mode (kicker on) could not easily be calculated from the continuous current and the duty cycle (1/2000 for $500 \mathrm{~ns}$ pulses at $1 \mathrm{kHz}$ ). Instead, the beam current was measured both in continuous and pulsed mode whenever the latter was within the range of the CI. For lower, pulsed currents the intensity was extrapolated from the continuous one and the measured ratio of both for data points of the same series, with correspondingly larger uncertainties. Note, that the CI does not reproduce the pulsed beam structure, but represents a mean current instead. Additional beam current measurements have been carried out with previously calibrated radiochromic films as described at the end of section III-B, confirming the values obtained from the CI up to $4.1 \%$.

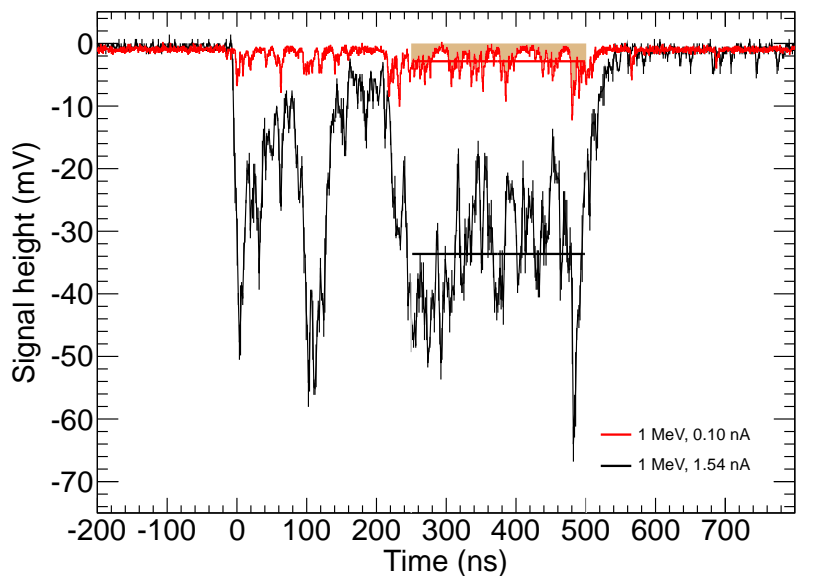

Fig. 11. (color on-line) Oscilloscope signals of two proton bunches for $1.0 \mathrm{MeV}$ beam energy and different beam intensities. These samples have been obtained without optical filters. The straight lines indicate mean pulse heights within a relatively stable interval (shaded area for the $0.1 \mathrm{nA}$ continuous current case). The two peaks at $t<200 \mathrm{~ns}$ are artefacts of the beam kicker. A $100 \mathrm{~ns}$ long interval outside the main pulse has been used to determine the zero level.

Data have been taken with beams of $1.0 \mathrm{MeV}$ and $1.5 \mathrm{MeV}$ protons. At $1.0 \mathrm{MeV}$, the continuous beam currents ranged from $42 \mathrm{pA}$ to $170 \mathrm{nA}$, and pulse heights have been measured without optical filter and with neutral density filters of OD1-3. At $1.5 \mathrm{MeV}$, a continuous beam interval of $200 \mathrm{pA}$ to $390 \mathrm{nA}$ was covered and filters of OD1-3 have been applied. Within each data subset (i.e., with the same beam energy and optical filter), the beam intensity on the scintillator has been varied through the collimator aperture. For each beam current, we have recorded several (typically 5-10) oscilloscope signals of single bunches, similar to those of Fig. 11. Note, that at the lowest intensities single proton peaks can be distinguished within the $500 \mathrm{~ns}$ bunch length. At higher intensities the individual pulses overlap, but the oscillations around the mean signal level may be large.

In order to obtain the absolute number of protons per time interval, we have started from the current measurements in pulsed mode, taking into account the time structure of the bunches. More precisely, we suppose that at $1 \mathrm{kHz}$ pulse rate one bunch carries $10^{-3}$ times the charge accumulated in 1 second on the CI. For several oscilloscope pulses the total area, $A_{t}$, and the area under an approximately constant interval at large signal height, $A_{s}$, have been calculated. In the examples of Fig. 11, the useful signal ranges from 250 to 500 ns, while 
the entire bunch interval is $[-50,550] \mathrm{ns}$. The area under the zero level, $A_{0}$, has been measured outside the main pulse $([-180,-80]$ ns in Fig. 11) and subtracted via

$$
A_{s}^{\prime}=A_{s}-\frac{T_{s}}{T_{0}} A_{0}, A_{t}^{\prime}=A_{t}-\frac{T_{t}}{T_{0}} A_{0}
$$

where $T_{s}, T_{t}$, and $T_{0}$ indicate the length of the signal, overall bunch, and zero level intervals, respectively. The number of protons in the signal interval is then supposed to correspond to the proportional part of the bunch charge, $A_{s}^{\prime} / A_{t}^{\prime}$. We have obtained consistent values for $A_{s}^{\prime} / A_{t}^{\prime}$ for a major number of pulses clearly below the PMT saturation level. The absolute proton numbers have been normalised to a $100 \mathrm{~ns}$ time interval for convenience.

The interval $T_{s}$ has also been used to determine the mean signal height, $U_{p}$ (horizontal bars in Fig. 11). Here, too, the mean signal height of the zero level interval has been subtracted. This procedure has been carried out for all recorded pulses at the same beam current. The mean values of each subset are represented in Figs. 12 and 13 as a function of protons per $100 \mathrm{~ns}$. The data points are measured data, with colours corresponding to different neutral density filters. They have been fitted by a linear function with slope, $m$, and an additional factor, $\alpha$, to account for saturation,

$$
U_{p}=\frac{m N_{p}}{1+\alpha N_{p}} .
$$

For those series, where all points are far below the saturation level, the parameter $\alpha$ has been set to 0 . The dashed lines represent the same fit results scaled with the inverse filter factor (i.e., a factor of $10(100,1000)$ for OD1 (OD2, OD3), respectively). The fit results for the slope and saturation parameter are given in Table [

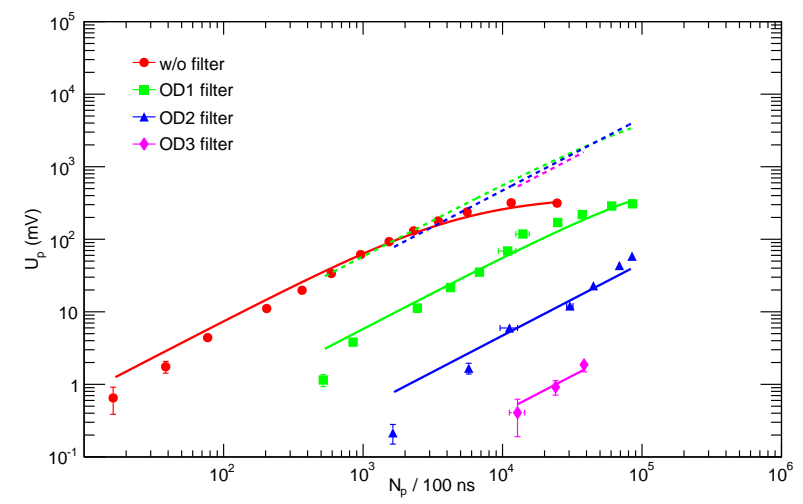

Fig. 12. (color on-line) Mean signal level on the oscilloscope as a function of the beam intensity, for $1.0 \mathrm{MeV}$ beam energy and different optical filters. Symbols and solid curves are measured data and fits following Eq. (4); dashed curves are the same fits multiplied by the inverse filter factor.

At $1.0 \mathrm{MeV}$, starting from the lowest beam currents (protons per bunch) without optical filter, we find a linear relation between signal height and particle numbers up to $2 \times 10^{3}$ protons/100 ns where, at more than $100 \mathrm{mV}$ pulse amplitude $U_{p}$, the onset of PMT saturation can be observed (Fig. 12). For the data points with OD1 filter, we see a similar relation with

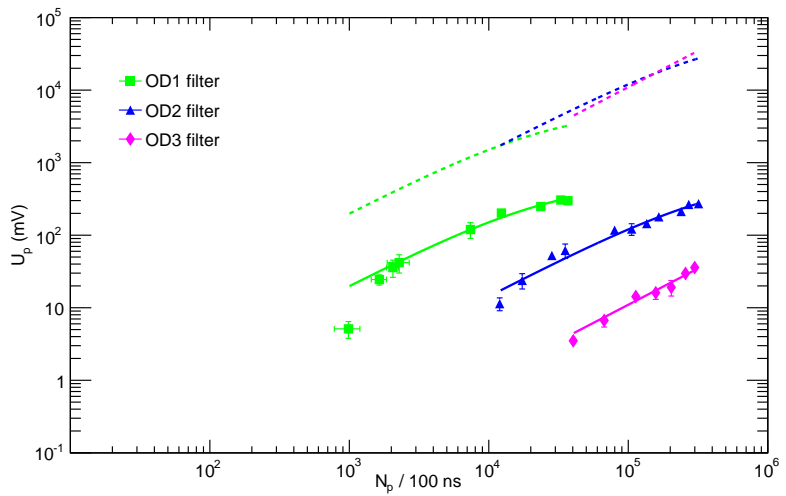

Fig. 13. (color on-line) Mean signal level on the oscilloscope as a function of the beam intensity, for $1.5 \mathrm{MeV}$ beam energy and different optical filters (see Fig. 12,

TABLE I

FIT RESULTS FOR THE SLOPES AND SATURATION PARAMETERS AT 1.0 AND 1.5 MEV. VALUES MARKED WITH STARS HAVE BEEN MULTIPLIED BY $10^{*}, 100^{* *}$, AND $1000^{* * *}$, RESPECTIVELY.

\begin{tabular}{clclc}
\hline Filter & $\begin{array}{l}m_{1} \\
(1.0 \mathrm{MeV})\end{array}$ & $\begin{array}{c}\alpha_{1} \\
(1.0 \mathrm{MeV})\end{array}$ & $\begin{array}{l}m_{2} \\
(1.5 \mathrm{MeV})\end{array}$ & $\begin{array}{c}\alpha_{2} \\
(1.5 \mathrm{MeV})\end{array}$ \\
\hline none & 0.0752 & $1.906 \times 10^{-4}$ & & \\
OD1 & $0.0524^{*}$ & $5.212 \times 10^{-6}$ & $0.205^{*}$ & $3.623 \times 10^{-5}$ \\
OD2 & $0.0470^{* *}$ & 0.0 & $0.146^{* *}$ & $2.194 \times 10^{-6}$ \\
OD3 & $0.0415^{* * *}$ & 0.0 & $0.110^{* * *}$ & 0.0 \\
\hline
\end{tabular}

saturation at higher beam currents. When multiplied by the inverse filter factor (10), the OD1 data approximately continue the linear trend of those without filter. A similar behaviour is observed for the data with OD2 and OD3 filters which, again after proper scaling, are consistent with the former ones. This is confirmed by the fit parameters (Table I), where the slopes range from 0.0415 to 0.0752 (after scaling), i.e. they coincide approximately within a factor 2 (mean value $\left.\bar{m}_{1}=0.0555 \pm 0.0149\right)$. The OD2 and OD3 series do not reach the saturation level. They could not be extended to higher proton numbers, due to limitations of the beam current from the tandem accelerator.

The data at $1.5 \mathrm{MeV}$ show a similar, overall consistency between series of different optical filters (Fig. 13). Here, PMT saturation is observed with OD1 and OD2 filters, while the OD3 series remains unsaturated even at the highest beam currents. The quantitative concordance is similar to the $1.0 \mathrm{MeV}$ data, with a mean value $\bar{m}_{2}=0.1538 \pm 0.0480$. The total range of protons per $100 \mathrm{~ns}$ interval has been extended to higher values with a maximum of $3 \times 10^{5}$. Again, the limiting factor preventing us from testing even larger particle numbers has been the available beam current.

The protons are stopped inside the scintillator, thus for equal particle numbers the measured pulse heights should be approximately proportional to the proton energy. This consistency between data of 1 and $1.5 \mathrm{MeV}$ can be checked by comparing the fitted slopes. Using the mean values of all series, one finds a ratio $\bar{m}_{2} / \bar{m}_{1}=2.77 \pm 1.14$, which is larger than the expected value (1.5). Note, that if energy loss of the protons in the mylar protection is taken into account, being 
more prominent for $1.0 \mathrm{MeV}$ protons than those of $1.5 \mathrm{MeV}$, the calculated ratio of energy depositions in the scintillator is approximately 1.66. Further, at the Bragg peak the amplitude is attenuated due to localised quenching. In this regime, the light output may thus be proportional to the path length rather than the particle energy, and hence the ratio of pulse heights should be approximately 2 . The experimental result is not sufficient to strictly support the expected proportionality with the proton energy or the path length within the scintillator. In order to translate a continuous time-of-flight signal of laseraccelerated protons into a quantitative spectrum, a repetition of the described measurements at higher proton energies (4$10 \mathrm{MeV}$ ) would be desirable, but was not possible within the scope of this work.

\section{Discussion AND CONCLUSIONS}

We have studied the response of two particle detectors to proton beams with energies up to $5.6 \mathrm{MeV}$. For radiochromic film of type HD-V2, we have obtained a calibration curve relating the optical density with the total deposited energy per area. Very consistent results have been found for protons of 1.5 and $4.0 \mathrm{MeV}$, for both continuous and pulsed beams. The validity of this calibration has been studied in various performance tests to simulate the response of RCF stacks to protons of single or multiple energies, including beam energies different to those applied for the calibration. Furthermore, we have demonstrated the capability of reconstructing the total deposited charge (equivalent to the particle number at fixed energy) for very non-uniform spatial distributions.

For the analysis of proton spectra under real-time conditions, we have designed an online monitor. We have demonstrated its capacity to detect single charged particles, including protons with energies as low as $0.46 \mathrm{MeV}$, with a very short range inside the active material. For the simultaneous detection of large numbers of particles, saturation of the PMT output voltage becomes important. Our setup includes variable optical filters to avoid PMT saturation and expand the dynamic range over several orders of magnitude. With pulsed beams from a tandem accelerator, we have shown stable signal levels up to $200 \mathrm{mV}$ for $500 \mathrm{~ns}$ long proton bunches, much more than required for time-of-flight measurements of laser-accelerated particles. Below the saturation level, the signal amplitude is proportional to the proton beam current. In addition, we have demonstrated consistency within a factor 2 in signal levels between data series obtained with different filters. At beam energies of 1 and $1.5 \mathrm{MeV}$, we have thus represented particle numbers as high as $3 \times 10^{5}$ in a $100 \mathrm{~ns}$ time interval. This upper limit was due to the maximum available beam current of the tandem accelerator. An extension to even higher intensities by at least two orders of magnitude, with filters of optical densities higher than OD3, would allow for studying saturation effects of the scintillator. In this case, the use of optical filters would not guarantee any more a linear relation between beam current and signal level.

The observed width of single-particle pulses (6 ns) is larger than expected from the intrinsic decay time of the scintillator (2.4 ns) and may limit the energy resolution of protons between 10 and $15 \mathrm{MeV}$ at 1.5-2 $\mathrm{m}$ target distance. The performance may be improved by a faster scintillator material, readout by a PMT with less transit time spread, and a better fiber-optic coupling. We plan to study these options, as well as alternatives such as a flexible adaption of the flight path, for the precise measurement of the energy spectra of laseraccelerated protons.

\section{ACKNOWLEDGMENTS}

The authors appreciate the use of the CNA installations and the contributions on behalf of the technical staff.

\section{REFERENCES}

[1] H. Daido, M. Nishiuchi, and A.S. Pirozhkov, "Review of laser-driven ion sourcesand their applications", Rep. Prog. Phys. 75, 056401 (2012).

[2] A. Macchi, M. Borghesi, and M. Passoni, "Ion acceleration by superintense laser-plasma interaction”, Rev. Mod. Phys. 85, 751-793 (2013).

[3] K.W.D. Ledingham and W. Galster, "Laser-driven particle and photon beams and some applications", New Journal of Physics 12, 045005 (2010).

[4] P.R. Bolton et al., "Instrumentation for diagnostics and control of laseraccelerated proton (ion) beams", Physica Medica 30, 255-270 (2014).

[5] D.S. Hey et al., "Use of gafchromic film to diagnose laser generated proton beams", Rev. Sci. Inst. 79, 053501 (2008).

[6] F. Nürnberg et al., "Radiochromic film imaging spectroscopy of laseraccelerated proton beams", Rev. Sci. Inst. 80, 033301 (2009).

[7] K. Harres et al., "Development and calibration of a Thomson parabola with microchannel plate for the detection of laser-accelerated $\mathrm{MeV}$ ions", Rev. Sci. Inst. 79, 093306 (2008).

[8] D.C. Carroll et al., "A modified Thomson parabola spectrometer for high resolution multi-MeV ion measurements - application to laser-driven ion acceleration", Nucl. Inst. Meth. A 620, 23-27 (2010).

[9] S. Reinhardt, W. Draxinger, J. Schreiber, and W. Assmann, "A pixel detector system for laser-accelerated ion detection", J. Inst. 8, P03008 (2013).

[10] S. Nakamura et al., "Real-time optimization of proton production by intense short-pulse laser with time-of-flight measurement", Jpn. J. Appl. Phys. 45, L913-L916 (2006).

[11] I.W. Choi et al., "Ion spectrometer composed of time-of-flight and Thomson parabola spectrometers for simultaneous characterization of laser-driven ions", Rev. Sci. Inst. 80, 053302 (2009).

[12] J.S. Green et al., "Scintillator-based ion beam profiler for diagnosing laser-accelerated ion beams", Proc. SPIE 8079, 807919 (2011).

[13] J. Metzkes et al., "A scintillator-based online detector for the angularly resolved measurement of laser-accelerated proton spectra", Rev. Sci. Inst. 83, 123301 (2012).

[14] D. Margarone et al., "Full characterization of laser-accelerated ion beams using Faraday cup, silicon carbide, and single-crystal diamond detectors", J. Appl. Phys. 109, 103302 (2011).

[15] P. Antici et al., "Energetic protons generated by ultrahigh contrast laser pulses interacting with ultrathin targets", Phys. Plasmas 14, 030701 (2007).

[16] Y. Fukuda et al., "Energy increase in multi-MeV ion acceleration in the interaction of a short pulse laser with a cluster-gas target", Phys. Rev. Lett. 103, 165002 (2009).

[17] A. Yogo et al., "Laser prepulse dependency of proton-energy distributions in ultraintense laser-foil interactions with an online time-of-flight technique", Phys. Plasmas 14, 043104 (2007).

[18] G.F. Knoll, "Radiation detection and measurement", 4th edition, John Wiley and Sons, 2010.

[19] N. Sinenian et al., "The response of CR-39 nuclear track detector to 1-9 MeV protons", Rev. Sci. Instrum. 82, 103303 (2011); Rev. Sci. Instrum. 85, 119901 (2014) (E).

[20] R. Dreindl, D. Georg, and M. Stock, "Radiochromic film dosimetry: Considerations on precision and accuracy for EBT2 and EBT3 type films", Z. Med. Phys. 24, 153-163 (2014).

[21] J. Kaufman et al., "Radiochromic film diagnostics for laser-driven ion beams", Proc. of SPIE 9515, 95151J-1 (2015).

[22] Information provided by the manufacturer/distributor (private communication). Note that an active layer thickness of $8 \mu \mathrm{m}$ has been stated incorrectly in some commercial documentation. 
[23] P. Bellido, M. Seimetz et al., "Dosimetric calibration of radiochromic film for laser-accelerated proton beams", 2013 IEEE Nuclear Science Symposium Conference Record.

[24] J. Ziegler and J. Biersack, "SRIM - The stopping range of ions in solids", Pergamon, 1985. http://www.srim.org/

[25] A. Piermattei et al., "Radiochromic film dosimetry of a low energy proton beam", Med. Phys. 7, 1655-1660 (2000).

[26] W.R. Leo, "Techniques for nuclear and particle physics experiments: A how-to approach", 2nd edition, Springer, New York, 1994.

[27] M. Seimetz, P. Bellido et al., "Time-of-flight detector for the characterisation of laser-accelerated protons", 2013 IEEE Nuclear Science Symposium Conference Record.

[28] Berkeley Nucleonics Corporation, http://www.berkeleynucleonics.com/ products/model-6010.html. 